\title{
The Passage of Lactic Acid Bacteria from Silage into Rumen Fluid, In Vitro Studies
}

\author{
Z. G. Weinberg, Y. Chen, and M. Gamburg \\ Forage Preservation and By-Products Research Unit, \\ Department of Food Science, Agricultural Research Organization, \\ The Volcani Center, Bet Dagan 50250, Israel
}

\section{ABSTRACT}

Inoculated silages sometimes improve cattle performance, possibly because of probiotic effects of lactic acid bacteria (LAB) silage inoculants. The cause of improved animal performance following feeding with inoculated silage is unclear. One issue in studying this phenomenon is to find out whether LAB pass from silage into the rumen fluid and survive in it. The purpose of the present study was to determine whether LAB from inoculated and uninoculated silages pass into the rumen fluid in vitro. Wheat and corn silages, uninoculated or inoculated with 1 of 10 commercial silage inoculant LAB, were prepared in glass jars. After ensiling, a 2.5-g silage sample was added to $25 \mathrm{~mL}$ of heat-sterilized or strained rumen fluid together with $5 \mathrm{~g} / \mathrm{L}$ glucose, and incubated for 48 $\mathrm{h}$ at $39^{\circ} \mathrm{C}$. Analysis of the incubated rumen fluid included $\mathrm{pH}$ measurement, enumeration of LAB, and determination of lactic acid and volatile fatty acids (VFA). The $\mathrm{pH}$ of the rumen fluid decreased during incubation; both heat-sterilized and strained rumen fluid contained large numbers of LAB. The heat-sterilized rumen fluid contained lactic acid in addition to VFA, whereas the strained rumen fluid contained only VFA. The results indicate that LAB pass from silage samples into the rumen fluid in vitro and survive there. Their interactions with rumen microorganisms should be studied further to understand how some silage inoculant LAB exhibit probiotic effects in dairy cattle.

(Key words: lactic acid bacteria silage inoculants, rumen fluid, probiotic effect)

Abbreviation key: ARF = heat-sterilized (autoclaved) rumen fluid; $\mathbf{L A B}$ = lactic acid bacteria; $\mathbf{R F}=$ rumen fluid; $\mathbf{S R F}$ = strained rumen fluid.

\section{INTRODUCTION}

Silage, which is forage preserved through lactic acid fermentation, is a major component in the rations of

Received April 15, 2004.

Accepted June 16, 2004.

Corresponding author: Zwi G. Weinberg; e-mail: zgw@volcani. agri.gov.il. dairy cattle. Inoculants comprising mainly lactic acid bacteria (LAB) are used as silage additives to enhance the ensiling fermentation. An inoculation rate of $10^{5}$ to $10^{6}$ microorganisms per gram of crop is often sufficient for the inoculant LAB to overwhelm the epiphytic LAB and become the predominant population in the silage (Weinberg and Muck, 1996; Kung et al., 2003).

In some cases, feeding with LAB-treated silage has been observed to affect animal performance; in 25 to $40 \%$ of the reviewed studies, feed intake, weight gain, feed efficiency, and/or milk production were improved, and the improvements ranged from 5 to $11 \%$ (Muck, 1993; Kung et al., 2003).

A considerable number of cattle-feeding experiments, in which moist grass silage was treated with a single silage inoculant strain, Lactobacillus plantarum MTD1, were performed in Northern Ireland (Keady et al., 1994; Keady and Steen, 1994, 1995). The majority of these studies found that silages inoculated with this strain improved animal performance regardless of fermentation quality. When the inoculant was added to silage immediately before feeding, there were no significant effects on digestibility of DM, nitrogen, NDF, or modified $\mathrm{ADF}$ (Keady and Steen, 1996), which might suggest that the benefits resulted from the silage fermentation rather than from effects of the LAB in the rumen itself. In contrast, in a recent study (Khuntia and Chaudhary, 2002), dietary addition of a mixed culture of LAB increased DM intake, weight gain, and DM digestibility in calves. Their rumen $\mathrm{pH}$ was lower and lactic acid concentration was higher following LAB feeding. Salawu et al. (2001) found that application of L. plantarum to pea-wheat silage increased the rate of nitrogen and NDF degradation in the rumen. Malik and Sharma (1998) inoculated rumen fluid with various microorganisms in the presence of wheat straw and concentrates, and found that L. acidophilus improved DM and OM digestibility in vitro, compared with an untreated control.

The cause of the improvement in animal performance following feeding with inoculated silage is unclear, but the results of feeding experiments suggest a possible probiotic effect of the LAB used in inoculants. One hypothesis is that certain LAB strains interact with rumen 
microorganisms to enhance rumen functionality and animal performance. Such a hypothesis is consistent with Fuller's (1989) definition of a probiotic: "Live microbial feed supplement which beneficially affects the host animal by improving its intestinal microbial balance" (Fuller, 1989). To affect rumen microflora, LAB ingested by the animals along with the silage would have to survive under rumen conditions.

A previous study indicated that freeze-dried cultures of LAB used in silage inoculants survived in rumen fluid; the $\mathrm{pH}$ of strained rumen fluid treated with $\mathrm{LAB}$ cultures was generally higher than that of uninoculated control rumen fluid throughout the 72- to 96-h incubation period (Weinberg et al., 2003, 2004).

The purpose of the present study was to determine whether LAB from inoculated silages pass into the rumen fluid, and to examine the effects of inoculated silages on the rumen fluid in vitro.

\section{MATERIALS AND METHODS}

\section{Ensiling}

Wheat at the milk ripening stage (DM $350 \mathrm{~g} / \mathrm{kg}$ ) and corn at the early dent stage (DM $190 \mathrm{~g} / \mathrm{kg}$ ) and at the half-milk line (DM $280 \mathrm{~g} / \mathrm{kg}$ ) were chopped and ensiled in sealed 0.25 -L glass jars. There were 4 jars per treatment. Silage treatments included control (no additives) and addition of 1 of $10 \mathrm{LAB}$ silage inoculants, which were applied at $10^{6} \mathrm{cfu} / \mathrm{g}$.

The wheat and corn silages were stored at room temperature $\left(25\right.$ to $28^{\circ} \mathrm{C}$ ) for 8 and $4 \mathrm{mo}$, respectively, after which the silages were analyzed for $\mathrm{pH}, \mathrm{LAB}$ numbers, lactic acid, and volatile fermentation end-products.

\section{Inoculants}

The following commercial inoculants for silage were used: Lactobacillus plantarum MTD1 (Ecosyl, Yorkshire, UK); Pediococcus pentosaceus (Ecosyl, Yorkshire, UK); L. plantarum (Agri-King, Fulton, IL); L. pentosus (Agri-King, Fulton, IL); Pediococcus pentosaceus (AgriKing, Fulton, IL); Enterococcus faecium (C) (Agri-King, Fulton, IL); E. faecium (Q) (Agri-King, Fulton, IL); $L$. buchneri (Biotal Canada Limited, Calgary, AB, Canada); 11A44 Pioneer containing L. buchneri (Pioneer Hi-Bred International, Inc., Johnston, IA); and 1188 Pioneer containing L. plantarum and E. faecium (Pioneer Hi-Bred International, Inc., Des Moines, IA).

The numbers of LAB cells in the dry products were determined before the experiments by suspending the inoculants in deionized water and pour plating serial dilutions into Rogosa or De Man, Rogosa, and Sharpe agar (Oxoid Ltd., Basingstoke, UK). De Man, Rogosa, and Sharpe agar was used for all products that contained
E. faecium. The inoculants were applied by suspending an adequate weight (according to the LAB number in the product) in $20 \mathrm{~mL}$ of water and spraying over $2 \mathrm{~kg}$ of chopped forage.

\section{Experiments with Rumen Fluid}

Rumen fluid (RF) was collected from 2 fistulated Holstein dry cows that were fed on $6 \mathrm{~kg}$ of wheat hay and $4 \mathrm{~kg}$ DM of TMR containing 30\% concentrated grains, $35 \%$ wheat and corn silage, $15 \%$ soybean and sunflower meal, and $20 \%$ by-products (cotton seed, wheat bran, and gluten feed) and supplemental vitamins and minerals. The RF was strained through 4 layers of gauze and used as strained RF (SRF), or it was set for $1 \mathrm{~h}$ in Imhoff cones after which the middle layer was heat-sterilized (autoclaved) for $15 \mathrm{~min}$ at $121^{\circ} \mathrm{C}$ (ARF).

A 2.5-g pooled sample (wet weight) of each silage treatment was added in duplicates to $25 \mathrm{~mL}$ of SRF or ARF, and sterile $50 \%$ (wt/vol) glucose solution was added, to a final concentration of $5 \mathrm{~g} / \mathrm{L}$, as an available energy source for the LAB. The tubes with the RF and silage samples were flushed with $\mathrm{CO}_{2}$, sealed with rubber stoppers and shaken at $39^{\circ} \mathrm{C}$. In the trials with corn silages, $\mathrm{RF}$ (SRF and ARF) samples without added silage served as controls. Two tubes from each treatment were sampled after 24 and $48 \mathrm{~h}$ and the samples were analyzed for $\mathrm{pH}, \mathrm{LAB}$ numbers, and VFA. Experiments for each crop were performed in 2 separate batches for inoculants 1 to 5 and for inoculants 6 to 10 (plus control silages). Thus, there were 6 experiments with 6 different batches of RF.

\section{Analyses}

Dry matter was determined by oven drying for $48 \mathrm{~h}$ at $60^{\circ} \mathrm{C}$. Lactic acid was determined spectrophotometrically, according to Barker and Summerson (1941). Volatile fermentation products in the silages and VFA in the RF were determined by gas chromatography with a semicapillary FFAP column (Hewlett Packard, Waldbronn, Germany) over a temperature range of 40 to $230^{\circ} \mathrm{C}$.

The enumeration of LAB was performed with pour plates in Rogosa agar (Oxoid). De Man, Rogosa, and Sharpe agar (Oxoid) was used in the experiments with corn for the initial RF, control silages, and silages inoculated with Enterococcus spp. Plates were incubated at $30^{\circ} \mathrm{C}$ for $3 \mathrm{~d}$.

Statistical analysis of the RF samples included ANOVA and Duncan's multiple range test using the GLM procedure of SAS (SAS Institute, Inc., Cary, NC). The analysis was applied for each experiment (batch of RF) separately, and in each experiment separately for ARF 
Table 1. $\mathrm{pH}$ and fermentation products of the wheat silages (DM $350 \mathrm{~g} / \mathrm{kg}$ ). Fermentation products are given in $\mathrm{g} / \mathrm{kg}(\mathrm{DM})$ and $\mathrm{LAB}$ in $\log 10 \mathrm{cfu} / \mathrm{g}(\mathrm{DM})$.

\begin{tabular}{llllll}
\hline Treatment & $\mathrm{pH}$ & Lactic acid & Ethanol & Acetic acid & LAB $^{1}$ \\
\hline Control & $4.5 \pm 0.01$ & $38 \pm 6$ & $13 \pm 1$ & $30 \pm 4$ & 7.0 \\
Lactobacillus plantarum MTD1 (Ecosyl) & 4.0 & 33 & 13 & 10 & 6.7 \\
Pediococcus pentosaceus (Ecosyl) & 4.1 & 34 & 3 & 15 & 6.3 \\
L. plantarum (Agri-King) & 4.2 & 50 & $\mathrm{NF}^{2}$ & 13 & 6.1 \\
L. pentosus (Agri-King) & 3.9 & 64 & $\mathrm{NF}$ & 10 & 5.6 \\
P. pentosaceus (Agri-King) & 3.9 & 38 & 4 & 16 & 6.5 \\
Enterococcus faecium C (Agri-King) & 4.5 & 46 & 7 & 31 & 6.3 \\
E. faecium Q (Agri-King) & 4.0 & 39 & 3 & 19 & 7.0 \\
L. buchneri (Biotal) & 4.2 & 5 & 8 & 63 & 7.1 \\
L. buchneri 11A44 (Pioneer) & 4.3 & 27 & 6 & 46 & 6.5 \\
L. plantarum and E. faecium 1188 (Pioneer) & 3.9 & 29 & 13 & 17 & 6.7 \\
\hline
\end{tabular}

${ }^{1} \mathrm{LAB}=$ Lactic acid bacteria.

${ }^{2} \mathrm{NF}=$ Not found.

and SRF. The experimental design was randomized and the experimental units were the individual tubes containing $25 \mathrm{~mL}$ of $\mathrm{RF}$ with the various silage treatments. The statistical model tested the effects of the silage inoculant treatments on the dependent chemical variables that were measured ( $\mathrm{pH}$, lactic acid, and VFA). The standard errors were assessed from the root mean squared error for each variable.

\section{RESULTS}

Tables 1 to 3 summarize the analysis results of the silages that were treated with the various inoculants. Most silages contained large LAB populations $\left(\geq 10^{6} \mathrm{cfu} /\right.$ $\mathrm{g}$ of DM). The major fermentation product was lactic acid; substantial concentrations of acetic acid were also found, especially in silages inoculated with the heterofermentative L. buchneri (inoculants 8 and 9). In the early dent corn, the ratio of lactic: acetic acid ratio was smaller, and this may be attributed to an inadequate DM content.

Table 4 gives the analysis of the fresh RF. The $\mathrm{pH}$ of the ARF before silage addition ranged from 9.0 to 9.4; no $\mathrm{LAB}$ were found in the fresh $\mathrm{ARF}$ in any of the 6 trials. The $\mathrm{pH}$ of the SRF at the beginning of the experiments ranged from 6.4 to $7.0 ; \log _{10}(\mathrm{LAB} \mathrm{cfu} / \mathrm{mL})$ in $\mathrm{SRF}$ ranged from 2.6 to 5.5 .

Tables 5 to 10 give the analysis results of the ARF and SRF after incubation with silage samples. In all trials, the $\mathrm{pH}$ of the silage-added $\mathrm{RF}$ decreased during the incubation, and it was lower in the ARF than in the SRF. The LAB populations increased during incubation and were larger in the ARF than in the SRF. The $\mathrm{pH}$ of the ARF without silage addition remained higher than that of the silage-added ARF. In 2 trials with corn, very low LAB counts were found in the ARF without silage addition after incubation, possibly because of cross-contamination. The SRF without added silage had higher $\mathrm{pH}$ values than those of the silage-added $\mathrm{SRF}$, but in most cases, their LAB counts were lower.

Lactic acid and VFA were measured at the end of the incubation period, after $48 \mathrm{~h}$; this decision was based on results from a previous in vitro study (Weinberg et al., 2003) with freeze-dried LAB inoculants which survived in $\mathrm{RF}$ for $72 \mathrm{~h}$ and affected VFA in SRF even after 72

Table 2. $\mathrm{pH}$ and fermentation products of the early dent corn silages (DM $190 \mathrm{~g} / \mathrm{kg}$ ). Fermentation products are given in $\mathrm{g} / \mathrm{kg}(\mathrm{DM})$ and $\mathrm{LAB}$ in $\log _{10} \mathrm{cfu} / \mathrm{g}(\mathrm{DM})$.

\begin{tabular}{|c|c|c|c|c|c|}
\hline Treatment & $\mathrm{pH}$ & Lactic acid & Ethanol & Acetic acid & $\mathrm{LAB}^{1}$ \\
\hline Control & $4.3 \pm 0.01$ & $35 \pm 11$ & $10 \pm 4$ & $48 \pm 6$ & $8.7(7.3)^{2}$ \\
\hline Lactobacillus plantarum MTD1 (Ecosyl) & 4.2 & 36 & 9 & 37 & 8.6 \\
\hline Pediococcus pentosaceus (Ecosyl) & 4.3 & 29 & 14 & 48 & 8.7 \\
\hline L. plantarum (Agri-King) & 4.0 & 46 & 13 & 53 & 8.7 \\
\hline L. pentosus (Agri-King) & 4.1 & 43 & 12 & 50 & 8.7 \\
\hline P. pentosaceus (Agri-King) & 4.1 & 51 & 10 & 46 & 8.6 \\
\hline Enterococcus faecium C (Agri-King) & 4.4 & 57 & 13 & 27 & 7.4 \\
\hline E. faecium Q (Agri-King) & 4.2 & 43 & 10 & 46 & 7.6 \\
\hline L. buchneri (Biotal) & 3.8 & 36 & $\mathrm{NF}^{3}$ & 43 & 7.0 \\
\hline L. buchneri 11A44 (Pioneer) & 4.0 & 71 & 6 & 43 & 7.0 \\
\hline L. plantarum and E. faecium 1188 (Pioneer) & 4.1 & 60 & 7 & 39 & 7.6 \\
\hline
\end{tabular}

${ }^{1} \mathrm{LAB}=$ Lactic acid bacteria.

${ }^{2}$ Number in parentheses is from De Man, Rogosa, and Sharpe (MRS) agar.

${ }^{3} \mathrm{NF}=$ Not found. 
Table 3. $\mathrm{pH}$ and fermentation products of the half-milk line corn silages (DM $280 \mathrm{~g} / \mathrm{kg}$ ). Fermentation products are given in $\mathrm{g} / \mathrm{kg}(\mathrm{DM})$ and LAB in $\log _{10} \mathrm{cfu} / \mathrm{g}(\mathrm{DM})$.

\begin{tabular}{llllll}
\hline Treatment & $\mathrm{pH}$ & Lactic acid & Ethanol & Acetic acid & $\mathrm{LAB}^{1}$ \\
\hline Control & $3.6 \pm 0.07$ & $65 \pm 1$ & $8 \pm 2$ & $22 \pm 4$ & $5.8(6.7)^{2}$ \\
Lactobacillus plantarum MTD1 (Ecosyl) & 3.7 & 55 & 5 & 15 & 6.4 \\
Pediococcus pentosaceus (Ecosyl) & 3.6 & 64 & 7 & 22 & 6.8 \\
L. plantarum (Agri-King) & 3.6 & 66 & 6 & 19 & 6.1 \\
L. pentosus (Agri-King) & 3.6 & 77 & 5 & 17 & 6.5 \\
P. pentosaceus (Agri-King) & 3.6 & 69 & 5 & 19 & 6.3 \\
Enterococcus faecium C (Agri-King) & 3.7 & 84 & 7 & 20 & 6.7 \\
E. faecium Q (Agri-King) & 3.7 & 66 & 6 & 21 & 7.2 \\
L. buchneri (Biotal) & 3.8 & 37 & 5 & 27 & 7.1 \\
L. buchneri 11A44 (Pioneer) & 3.8 & 41 & 5 & 29 & 6.9 \\
L. plantarum and E. faecium 1188 (Pioneer) & 3.7 & 47 & 4 & 24 & 7.2 \\
\hline
\end{tabular}

${ }^{1} \mathrm{LAB}=$ Lactic acid bacteria.

${ }^{2}$ Number in parentheses is from De Man, Rogosa, and Sharpe (MRS) agar.

$\mathrm{h}$ of incubation. In the current experiments, lactic acid was found in the silage-added ARF in all trials except for the last trial with corn (Table 10). Some treatments had lower concentrations of lactic acid, for example, inoculants 8 and 9 (Tables 6 and 8), which contained the heterofermentative $L$. buchneri, which usually produces less lactic acid in silage (as can be seen in Tables 1 and 3). The SRF did not contain lactic acid, which was probably converted into VFA by the indigenous rumen microorganisms. Indeed, the total VFA concentration in the SRF was always higher than that in the ARF. In the $\mathrm{RF}$ with no added silage (both $\mathrm{ARF}$ and $\mathrm{SRF}$ ), no lactic acid was found and the total VFA concentration was usually lower than in the silage-added RF. In the $\mathrm{ARF}$, the apparent VFA production (48 $\mathrm{h}$ total VFA concentration minus their concentration in the corresponding fresh $\mathrm{RF}$ ) was negative in the experiments with wheat and early dent corn silages, and was positive in the experiments with half-milk line corn silages (in which low or no lactic acid was found). The size and sign of the apparent VFA production depends on VFA concentration in the fresh $\mathrm{RF}$, and in the experiments with the half-milk line corn silage, they were low (Table 4). In the SRF, the apparent VFA production was always positive. In the RF with no added silage (both $A R F$ and SRF), the apparent VFA production was usually lower than in the silage-added RF.

\section{DISCUSSION}

The current experiments were conducted as part of a broader research objective, namely to find out how LAB silage inoculants enhance ruminant performance. The first step in this project was to determine whether LAB

Table 4. $\mathrm{pH}$, lactic acid bacteria, and fermentation products in the fresh rumen fluid.

\begin{tabular}{|c|c|c|c|c|c|c|c|c|c|}
\hline \multirow[b]{2}{*}{ Experiment } & \multirow[b]{2}{*}{ Silage $^{1}$} & \multirow[b]{2}{*}{$\begin{array}{l}\text { Rumen } \\
\text { fluid }^{2}\end{array}$} & \multirow[b]{2}{*}{$\mathrm{pH}$} & \multirow[b]{2}{*}{$\mathrm{LAB}^{3}$} & \multirow[b]{2}{*}{$\begin{array}{l}\text { Lactic acid } \\
(\mathrm{m} M)\end{array}$} & \multirow[b]{2}{*}{$\begin{array}{l}\text { Total VFA } \\
(\mathrm{m} M)\end{array}$} & \multicolumn{3}{|c|}{ VFA mol/100 mol } \\
\hline & & & & & & & $\begin{array}{l}\text { Acetic } \\
\text { acid }\end{array}$ & $\begin{array}{l}\text { Propionic } \\
\text { acid }\end{array}$ & $\begin{array}{l}\text { Butyric } \\
\text { acid }\end{array}$ \\
\hline \multirow[t]{2}{*}{1} & \multirow[t]{2}{*}{ wheat } & $\mathrm{ARF}$ & 9.3 & $\mathrm{NF}^{5}$ & $<0.5$ & 101.1 & 69.0 & 22.1 & 8.8 \\
\hline & & $\mathrm{SRF}$ & 7.0 & 2.6 & $<0.5$ & 87.4 & 67.2 & 22.9 & 10.0 \\
\hline \multirow[t]{2}{*}{2} & \multirow[t]{2}{*}{ wheat } & $\mathrm{ARF}$ & 9.0 & $\mathrm{NF}$ & $<0.5$ & 122.3 & 68.4 & 22.6 & 8.9 \\
\hline & & SRF & 7.1 & 5.5 & $<0.5$ & 83.2 & 70.0 & 21.9 & 8.2 \\
\hline \multirow[t]{2}{*}{3} & \multirow[t]{2}{*}{ ED corn } & $\mathrm{ARF}$ & 9.0 & $\mathrm{NF}$ & $<0.5$ & 134.1 & 65.2 & 21.8 & 12.9 \\
\hline & & SRF & 6.4 & 5.3 & $<0.5$ & 136.3 & 66.2 & 21.5 & 12.3 \\
\hline \multirow[t]{2}{*}{4} & \multirow[t]{2}{*}{ ED corn } & $\mathrm{ARF}$ & 9.3 & $\mathrm{NF}$ & $<0.5$ & 98.6 & 67.4 & 21.8 & 10.8 \\
\hline & & SRF & 7.0 & 4.8 & $<0.5$ & 90.3 & 67.6 & 21.6 & 10.8 \\
\hline \multirow[t]{2}{*}{5} & \multirow[t]{2}{*}{ HM corn } & $\mathrm{ARF}$ & 9.4 & $\mathrm{NF}$ & $<0.5$ & 49.4 & 65.3 & 26.5 & 8.1 \\
\hline & & SRF & 7.0 & 5.1 & $<0.5$ & 91.0 & 66.8 & 24.1 & 9.1 \\
\hline \multirow[t]{2}{*}{6} & \multirow[t]{2}{*}{ HM corn } & $\mathrm{ARF}$ & 9.4 & $\mathrm{NF}$ & $<0.5$ & 64.6 & 68.1 & 22.1 & 9.8 \\
\hline & & SRF & 7.0 & 3.5 & $<0.5$ & 41.2 & 69.9 & 20.9 & 9.2 \\
\hline
\end{tabular}

${ }^{1} \mathrm{ED}$ corn = Early dent corn at $190 \mathrm{~g} / \mathrm{kg}$ DM; HM corn = half-milk line corn at $280 \mathrm{~g} / \mathrm{kg} \mathrm{DM}$.

${ }^{2} \mathrm{ARF}=$ Autoclaved (heat-sterilized) rumen fluid; $\mathrm{SRF}=$ strained rumen fluid.

${ }^{3} \mathrm{LAB}$ (lactic acid bacteria) are given as $\log _{10} \mathrm{cfu} / \mathrm{mL}$.

${ }^{4}$ Total VFA (volatile fatty acids) is the sum of acetate, propionate, and butyrate.

${ }^{5} \mathrm{NF}=$ Not found. 
Table 5. $\mathrm{pH},{ }^{1}$ lactic acid bacteria, ${ }^{2}$ and fermentation products ${ }^{3}$ in autoclaved (ARF) and strained (SRF) rumen fluid with wheat silages in the first experiment.

\begin{tabular}{|c|c|c|c|c|c|c|c|c|c|c|}
\hline \multirow[b]{2}{*}{ Rumen fluid/silage treatment } & \multirow[b]{2}{*}{$\mathrm{pH}-24$} & \multirow[b]{2}{*}{$\mathrm{pH}-48$} & \multirow[b]{2}{*}{ LAB-24 } & \multirow[b]{2}{*}{ LAB-48 } & \multirow{2}{*}{$\begin{array}{l}\text { Lactic } \\
\text { acid- } 48 \\
(\mathrm{~m} M)\end{array}$} & \multirow{2}{*}{$\begin{array}{l}\text { Total } \\
\text { VFA }\end{array}$} & \multirow{2}{*}{$\begin{array}{l}\text { Apparent } \\
\text { VFA } \\
\text { production }\end{array}$} & \multicolumn{3}{|c|}{ VFA $(\mathrm{mol} / 100 \mathrm{~mol})$} \\
\hline & & & & & & & & Acetate & Propionate & Butyrate \\
\hline \multicolumn{11}{|l|}{$\overline{\mathrm{ARF}}$} \\
\hline Control & 5.91 & $5.09^{a, b}$ & 7.6 & 7.6 & 56.4 & 88.5 & -12.6 & $72.3^{\mathrm{a}}$ & $20.5^{\mathrm{a}}$ & $7.1^{\mathrm{b}}$ \\
\hline Lactobacillus plantarum MTD1 (Ecosyl) & 5.36 & $5.27^{\mathrm{a}}$ & 8.2 & $>9.0$ & 62.8 & 86.7 & -14.4 & $69.2^{\mathrm{a}, \mathrm{b}}$ & $23.3^{\mathrm{a}}$ & $7.5^{\mathrm{b}}$ \\
\hline Pediococcus pentosaceus (Ecosyl) & 4.99 & $4.92^{\mathrm{b}, \mathrm{c}}$ & 8.1 & 7.7 & 75.1 & 92.8 & -8.3 & $71.6^{\mathrm{a}}$ & $21.8^{\mathrm{a}}$ & $6.6^{\mathrm{b}}$ \\
\hline L. plantarum (Agri-King) & 5.00 & $4.89^{b, c}$ & $>9.0$ & 7.6 & 77.6 & 82.4 & -18.7 & $71.0^{\mathrm{a}}$ & $22.1^{\mathrm{a}}$ & $6.9^{\mathrm{b}}$ \\
\hline L. pentosus (Agri-King) & 4.60 & $5.01^{b, c}$ & 8.0 & 7.4 & 69.5 & 99.1 & -2.0 & $58.8^{\mathrm{b}}$ & $16.0^{\mathrm{b}}$ & $25.1^{\mathrm{a}}$ \\
\hline P. pentosaceus (Agri-King) & 6.11 & $4.79^{\mathrm{c}}$ & 7.1 & 7.5 & 86.0 & 88.3 & -12.8 & $73.6^{\mathrm{a}}$ & $19.6^{\mathrm{a}, \mathrm{b}}$ & $6.7^{\mathrm{b}}$ \\
\hline Standard error & 0.47 & 0.09 & & & 11.7 & 12.7 & & 4.3 & 1.6 & 5.2 \\
\hline \multicolumn{11}{|l|}{ SRF } \\
\hline Control & $5.58^{\mathrm{b}}$ & $5.50^{\mathrm{a}}$ & 5.6 & 5.7 & $<0.5$ & $199.0^{\mathrm{b}, \mathrm{c}}$ & 111.6 & $35.6^{\mathrm{b}}$ & $29.3^{\mathrm{b}}$ & $32.2^{\mathrm{a}}$ \\
\hline L. plantarum MTD1 (Ecosyl) & $5.66 \mathrm{a}$ & $5.47^{\mathrm{a}}$ & 5.1 & 4.1 & $<0.5$ & $176.7^{\mathrm{c}}$ & 89.3 & $51.7^{\mathrm{a}}$ & $35.4^{\mathrm{a}}$ & $12.9^{\mathrm{b}}$ \\
\hline P. pentosaceus $(\mathrm{E} \cos \mathrm{l})$ & $5.54^{\mathrm{b}, \mathrm{c}}$ & $5.50^{\mathrm{a}}$ & 7.0 & 6.4 & $<0.5$ & $241.0^{\mathrm{a}, \mathrm{b}}$ & 153.6 & $42.2^{\mathrm{b}}$ & $34.3^{\mathrm{a}, \mathrm{b}}$ & $23.4^{\mathrm{a}, \mathrm{b}}$ \\
\hline L. plantarum (Agri-King) & $5.52^{\mathrm{c}}$ & $5.43^{\mathrm{a}, \mathrm{b}}$ & 7.3 & 6.9 & $<0.5$ & $259.7^{\mathrm{a}}$ & 172.3 & $40.0^{\mathrm{b}}$ & $38.1^{\mathrm{a}}$ & $21.9^{\mathrm{a}, \mathrm{b}}$ \\
\hline L. pentosus (Agri-King) & $5.35^{\mathrm{d}}$ & $5.34^{\mathrm{b}}$ & 7.4 & 7.0 & $<0.5$ & $235.0^{\mathrm{a}, \mathrm{b}}$ & 147.6 & $41.1^{\mathrm{b}}$ & $38.3^{\mathrm{a}}$ & $20.6^{\mathrm{a}, \mathrm{b}}$ \\
\hline P. pentosaceus (Agri-King) & $5.37^{\mathrm{d}}$ & $5.31^{\mathrm{b}}$ & 6.8 & 5.7 & $<0.5$ & $214.4^{\mathrm{a}, \mathrm{b}, \mathrm{c}}$ & 127.0 & $40.0^{\mathrm{b}}$ & $34.9^{\mathrm{a}, \mathrm{b}}$ & $20.9^{\mathrm{a}, \mathrm{b}}$ \\
\hline Standard error & 0.02 & 0.05 & & & & 21.7 & & 10.6 & 8.0 & 11.0 \\
\hline
\end{tabular}

a,b,c For each rumen fluid separately, within a column, means with different letters are significantly different $(P<0.05)$.

${ }^{1} \mathrm{pH}-24, \mathrm{pH}-48=\mathrm{pH}$ values after 24 and $48 \mathrm{~h}$ of incubation at $39^{\circ} \mathrm{C}$.

${ }^{2}$ Lactic acid bacteria (LAB) are given as $\log 10 \mathrm{cfu} / \mathrm{mL}$. LAB-24, LAB-48 = LAB after 24 and $48 \mathrm{~h}$ of incubation at $39^{\circ} \mathrm{C} ; \mathrm{Lactic}$ acid- $48 \mathrm{~m} M=\operatorname{lactic}$ acid concentration in $\mathrm{m} M$ after $48 \mathrm{~h}$ of incubation at $39^{\circ} \mathrm{C}$.

${ }^{3}$ Total VFA = sum of acetate, propionate, and butyrate in $\mathrm{m} M$. Apparent VFA production is $48 \mathrm{~h}$ total VFA minus time 0 VFA concentrations (mM). 
Table 6. $\mathrm{pH},{ }^{1}$ lactic acid bacteria, ${ }^{2}$ and fermentation products ${ }^{3}$ in autoclaved (ARF) and strained (SRF) rumen fluid with wheat silages in the second experiment.

\begin{tabular}{|c|c|c|c|c|c|c|c|c|c|c|}
\hline \multirow[b]{2}{*}{ Rumen fluid/silage treatment } & \multirow[b]{2}{*}{$\mathrm{pH}-24$} & \multirow[b]{2}{*}{$\mathrm{pH}-48$} & \multirow[b]{2}{*}{ LAB-24 } & \multirow[b]{2}{*}{ LAB-48 } & \multirow{2}{*}{$\begin{array}{l}\text { Lactic } \\
\text { acid-48 } \\
(\mathrm{m} M)\end{array}$} & \multirow{2}{*}{$\begin{array}{l}\text { Total } \\
\text { VFA }\end{array}$} & \multirow{2}{*}{$\begin{array}{l}\text { Apparent } \\
\text { VFA } \\
\text { production } \\
\end{array}$} & \multicolumn{3}{|c|}{ VFA $(\mathrm{mol} / 100 \mathrm{~mol})$} \\
\hline & & & & & & & & Acetate & Propionate & Butyrate \\
\hline \multicolumn{11}{|l|}{$\mathrm{ARF}$} \\
\hline Control & $5.08^{\mathrm{b}}$ & $5.10^{\mathrm{c}}$ & 9.0 & 8.5 & $49.0^{\mathrm{a}, \mathrm{b}, \mathrm{c}}$ & $120.3^{\mathrm{a}}$ & -2.0 & 68.5 & $20.2^{\mathrm{a}, \mathrm{b}}$ & 11.2 \\
\hline Enterococcus faecium C (Agri-King) & $5.65^{\mathrm{a}, \mathrm{b}}$ & $5.01^{\mathrm{c}}$ & 6.7 & 8.9 & $67.7^{\mathrm{a}, \mathrm{b}}$ & $120.1^{\mathrm{a}}$ & -2.2 & 66.5 & $27.0^{\mathrm{a}}$ & 6.4 \\
\hline E. faecium Q (Agri-King) & $5.65^{\mathrm{a}, \mathrm{b}}$ & $5.11^{\mathrm{c}}$ & 7.0 & 8.7 & $70.2^{\mathrm{a}}$ & $74.3^{\mathrm{b}}$ & -48.0 & 74.0 & $20.1^{\mathrm{a}, \mathrm{b}}$ & 5.9 \\
\hline Lactobacillus buchneri (Biotal) & $5.85^{\mathrm{a}, \mathrm{b}}$ & $5.37^{\mathrm{b}, \mathrm{c}}$ & 5.5 & 8.9 & $26.8^{\mathrm{a}, \mathrm{b}, \mathrm{c}}$ & $90.3^{\mathrm{a}, \mathrm{b}}$ & -32.0 & 79.4 & $15.6^{\mathrm{b}, \mathrm{c}}$ & 5.0 \\
\hline L. buchneri 11A44 (Pioneer) & $6.54^{\mathrm{a}}$ & $5.63^{\mathrm{b}}$ & 8.8 & 8.8 & $16.2^{\mathrm{c}}$ & $102.3^{\mathrm{a}, \mathrm{b}}$ & -20.0 & 66.1 & $12.3^{\mathrm{c}}$ & 23.4 \\
\hline \multicolumn{11}{|l|}{ L. plantarum and E. faecium } \\
\hline 1188 (Pioneer) & $5.89^{\mathrm{a}, \mathrm{b}}$ & $6.19^{\mathrm{a}}$ & 6.9 & 8.3 & $23.6^{\mathrm{b}, \mathrm{c}}$ & $80.5^{\mathrm{a}, \mathrm{b}}$ & -41.8 & 49.6 & $15.7^{\mathrm{b}, \mathrm{c}}$ & 34.5 \\
\hline Standard error & 0.4 & 0.2 & & & 17.7 & 16.6 & & 6.7 & 1.9 & 13.2 \\
\hline \multicolumn{11}{|l|}{ SRF } \\
\hline Control & $5.49^{\mathrm{b}}$ & 5.42 & 6.1 & 6.2 & $<0.5$ & $272.8^{\mathrm{a}}$ & 189.6 & $44.8^{\mathrm{a}, \mathrm{b}}$ & $30.8^{\mathrm{a}}$ & $24.4^{\mathrm{b}}$ \\
\hline E. faecium C (Agri-King) & $5.47^{\mathrm{b}, \mathrm{c}}$ & 5.38 & 6.7 & 9.0 & $<0.5$ & $294.1^{\mathrm{a}}$ & 210.9 & $46.5^{\mathrm{a}, \mathrm{b}}$ & $25.7^{\mathrm{b}}$ & $27.8^{\mathrm{a}, \mathrm{b}}$ \\
\hline E. faecium Q (Agri-King) & $5.57^{\mathrm{a}}$ & 5.41 & 7.2 & 7.7 & $<0.5$ & $269.4^{\mathrm{a}}$ & 186.0 & $42.8^{\mathrm{b}}$ & $25.7^{\mathrm{b}}$ & $31.5^{\mathrm{a}, \mathrm{b}}$ \\
\hline L. buchneri (Biotal) & $5.41^{\mathrm{c}}$ & 5.34 & 7.0 & 8.6 & $<0.5$ & $245.2^{\mathrm{a}, \mathrm{b}}$ & 161.8 & $50.4^{\mathrm{a}}$ & $24.2^{\mathrm{b}}$ & $25.3^{\mathrm{b}}$ \\
\hline L. buchneri 11A44 (Pioneer) & $5.49^{\mathrm{b}}$ & 5.38 & 6.2 & 6.4 & $<0.5$ & $263.2^{\mathrm{a}}$ & 179.8 & $46.2^{\mathrm{a}, \mathrm{b}}$ & $26.9^{b}$ & $26.8^{\mathrm{a}, \mathrm{b}}$ \\
\hline \multicolumn{11}{|l|}{ L. plantarum and E. faecium } \\
\hline 1188 (Pioneer) & $5.57^{\mathrm{a}}$ & 5.38 & 6.8 & 6.7 & $<0.5$ & $200.0^{\mathrm{b}}$ & 116.6 & $42.5^{\mathrm{b}}$ & $23.1^{\mathrm{b}}$ & $34.4^{\mathrm{a}}$ \\
\hline Standard error & 0.03 & 0.03 & & & & 23.7 & & 2.4 & 1.6 & 3.2 \\
\hline
\end{tabular}

${ }^{a, b, c}$ For each rumen fluid separately, within a column, means with different letters are significantly different $(P<0.05)$

${ }^{1} \mathrm{pH}-24, \mathrm{pH}-48=\mathrm{pH}$ values after 24 and $48 \mathrm{~h}$ of incubation at $39^{\circ} \mathrm{C}$.

${ }^{2}$ Lactic acid bacteria (LAB) are given as $\log 10 \mathrm{cfu} / \mathrm{mL}$. LAB-24, LAB- $48=\mathrm{LAB}$ after 24 and $48 \mathrm{~h}$ of incubation at $39^{\circ} \mathrm{C} ; \mathrm{Lactic}$ acid- $48 \mathrm{~m} M=\operatorname{lactic}$ acid concentration in $\mathrm{m} M$ after $48 \mathrm{~h}$ of incubation at $39^{\circ} \mathrm{C}$.

${ }^{3}$ Total VFA = sum of acetate, propionate, and butyrate in $\mathrm{m} M$. Apparent VFA production is $48 \mathrm{~h}$ total VFA minus time 0 VFA concentrations (mM). 
Table 7. $\mathrm{pH}^{1}{ }^{1}$ lactic acid bacteria, ${ }^{2}$ and fermentation products ${ }^{3}$ in autoclaved (ARF) and strained (SRF) rumen fluid with early dent corn silages in the third experiment.

\begin{tabular}{|c|c|c|c|c|c|c|c|c|c|c|}
\hline \multirow[b]{2}{*}{ Rumen fluid/silage treatment } & \multirow[b]{2}{*}{$\mathrm{pH}-24$} & \multirow[b]{2}{*}{$\mathrm{pH}-48$} & \multirow[b]{2}{*}{ LAB-24 } & \multirow[b]{2}{*}{ LAB- 48} & \multirow{2}{*}{$\begin{array}{l}\text { Lactic } \\
\text { acid-48 } \\
(\mathrm{m} M)\end{array}$} & \multirow{2}{*}{$\begin{array}{l}\text { Total } \\
\text { VFA }\end{array}$} & \multirow{2}{*}{$\begin{array}{l}\text { Apparent } \\
\text { VFA } \\
\text { production }\end{array}$} & \multicolumn{3}{|c|}{$\mathrm{VFA}(\mathrm{mol} / 100 \mathrm{~mol})$} \\
\hline & & & & & & & & Acetate & Propionate & Butyrate \\
\hline \multicolumn{11}{|l|}{$\mathrm{ARF}$} \\
\hline Control & $5.16^{\mathrm{a}}$ & $5.06^{\mathrm{b}}$ & 7.6 & 8.7 & 53.3 & $125.9^{\mathrm{a}}$ & -8.2 & 60.9 & 30.0 & $9.1^{\mathrm{b}}$ \\
\hline Lactobacillus plantarum MTD1 (Ecosyl) & $4.99^{\mathrm{b}}$ & $4.96^{\mathrm{b}}$ & 7.4 & 8.3 & 49.9 & $104.9^{\mathrm{a}, \mathrm{b}}$ & -29.2 & 62.0 & 28.3 & $9.7^{\mathrm{b}}$ \\
\hline Pediococcus pentosaceus (Ecosyl) & $5.10^{\mathrm{a}, \mathrm{b}}$ & $4.96^{\mathrm{b}}$ & 7.3 & 8.5 & $59.9^{4}$ & $94.0^{\mathrm{b}}$ & -40.1 & 61.5 & 29.1 & $9.3^{\mathrm{b}}$ \\
\hline L. plantarum (Agri-King) & $5.05^{\mathrm{a}, \mathrm{b}}$ & $4.89^{\mathrm{b}}$ & 7.3 & 8.3 & 55.7 & $116.9^{\mathrm{a}, \mathrm{b}}$ & -17.2 & 62.7 & 26.6 & $10.7^{\mathrm{a}, \mathrm{b}}$ \\
\hline L. pentosus (Agri-King) & $5.03^{\mathrm{a}, \mathrm{b}}$ & $4.84^{\mathrm{b}}$ & 7.6 & 8.6 & $61.8^{4}$ & $107.1^{\mathrm{a}, \mathrm{b}}$ & -27.0 & 65.3 & 24.0 & $10.6^{\mathrm{a}, \mathrm{b}}$ \\
\hline P. pentosaceus (Agri-King) & $5.01^{\mathrm{b}}$ & $5.01^{\mathrm{b}}$ & 7.5 & 8.2 & 41.8 & $133.4^{\mathrm{a}}$ & -0.7 & 61.2 & 29.4 & $9.3^{\mathrm{b}}$ \\
\hline $\mathrm{ARF}$ alone & & $6.39^{\mathrm{a}}$ & & $\mathrm{NF}^{5}$ & $<0.5$ & $80.5^{\mathrm{b}}$ & -53.6 & 62.1 & 25.2 & $12.4^{\mathrm{a}}$ \\
\hline Standard error & 0.05 & 0.51 & & & 16.3 & 12.9 & & 2.7 & 0.8 & 3.2 \\
\hline \multicolumn{11}{|l|}{$\mathrm{SRF}$} \\
\hline Control & 5.24 & $5.19^{\mathrm{b}, \mathrm{c}}$ & 6.3 & 7.0 & $<0.5$ & 218.9 & 82.6 & 44.2 & 24.9 & 30.9 \\
\hline L. plantarum MTD1 (Ecosyl) & 5.26 & $5.22^{\mathrm{b}}$ & 6.1 & 6.7 & $<0.5$ & 192.3 & 56.0 & 45.5 & 25.6 & 28.9 \\
\hline P. pentosaceus (Ecosyl) & 5.26 & $5.15^{\mathrm{c}}$ & 6.4 & 7.2 & $<0.5$ & 245.7 & 109.4 & 45.0 & 23.8 & 31.2 \\
\hline L. plantarum (Agri-King) & 5.26 & $5.18^{\mathrm{b}, \mathrm{c}}$ & 5.0 & 8.3 & $<0.5$ & 273.5 & 137.2 & 43.9 & 25.0 & 31.0 \\
\hline L. pentosus (Agri-King) & 5.30 & $5.19^{\mathrm{b}, \mathrm{c}}$ & 6.2 & 7.2 & $<0.5$ & 275.2 & 138.9 & 44.5 & 25.6 & 29.9 \\
\hline P. pentosaceus (Agri-King) & 5.28 & $5.17^{\mathrm{c}}$ & 7.2 & 6.6 & $<0.5$ & 227.6 & 91.3 & 41.8 & 24.0 & 34.2 \\
\hline SRF alone & & $5.31^{\mathrm{a}}$ & & 7.5 & $<0.5$ & 186.4 & 50.1 & 50.3 & 23.8 & 26.0 \\
\hline Standard error & 0.02 & 0.02 & & & & 42.2 & & 7.6 & 4.1 & 3.6 \\
\hline
\end{tabular}

a,b,c For each rumen fluid separately, within a column, means with different letters are significantly different $(P<0.05)$.

${ }^{1} \mathrm{pH}-24, \mathrm{pH}-48=\mathrm{pH}$ values after 24 and $48 \mathrm{~h}$ of incubation at $39^{\circ} \mathrm{C}$.

${ }^{2}$ Lactic acid bacteria (LAB) are given as $\log 10 \mathrm{cfu} / \mathrm{mL}$. LAB-24, LAB-48 = LAB after 24 and $48 \mathrm{~h}$ of incubation at $39^{\circ} \mathrm{C} ; \mathrm{Lactic}$ acid- $48 \mathrm{~m} M=\operatorname{lactic}$ acid concentration in $\mathrm{m} M$ after $48 \mathrm{~h}$ of incubation at $39^{\circ} \mathrm{C}$

${ }^{3}$ Total VFA $=$ sum of acetate, propionate, and butyrate in $\mathrm{m} M$. Apparent VFA production is $48 \mathrm{~h}$ total VFA minus time 0 VFA concentrations (m $M$ )

${ }^{4}$ Large differences between duplicates.

${ }^{5} \mathrm{NF}=$ Not found 
Table 8. $\mathrm{pH},{ }^{1}$ lactic acid bacteria, ${ }^{2}$ and fermentation products $^{3}$ in autoclaved (ARF) and strained (SRF) rumen fluid with early dent corn silages in the fourth experiment.

\begin{tabular}{|c|c|c|c|c|c|c|c|c|c|c|}
\hline \multirow[b]{2}{*}{ Rumen fluid/silage treatment } & \multirow[b]{2}{*}{ pH-24 } & \multirow[b]{2}{*}{$\mathrm{pH}-48$} & \multirow[b]{2}{*}{ LAB-24 } & \multirow[b]{2}{*}{ LAB- 48} & \multirow{2}{*}{$\begin{array}{l}\text { Lactic } \\
\text { acid- } 48 \\
(\mathrm{~m} M)\end{array}$} & \multirow{2}{*}{$\begin{array}{l}\text { Total } \\
\text { VFA }\end{array}$} & \multirow{2}{*}{$\begin{array}{l}\text { Apparent } \\
\text { VFA } \\
\text { production }\end{array}$} & \multicolumn{3}{|c|}{ VFA (mol/100 mol) } \\
\hline & & & & & & & & Acetate & Propionate & Butyrate \\
\hline \multicolumn{11}{|l|}{$\mathrm{ARF}$} \\
\hline Control & $5.06^{\mathrm{a}, \mathrm{b}}$ & $4.97^{\mathrm{b}}$ & 8.8 & $8.9(8.7)^{4}$ & $65.3^{\mathrm{a}, 5}$ & 76.0 & -22.6 & $67.6^{\mathrm{a}, \mathrm{b}}$ & $23.9^{\mathrm{a}, \mathrm{b}}$ & $8.5^{\mathrm{b}, \mathrm{c}}$ \\
\hline Enterococcus faecium C (Agri-King) & $5.00^{\mathrm{b}}$ & $4.97^{\mathrm{b}}$ & 8.3 & 8.5 & $55.6^{\mathrm{a}, \mathrm{b}}$ & 84.2 & -14.4 & $69.7^{\mathrm{a}}$ & $22.0^{\mathrm{b}, \mathrm{c}}$ & $8.3^{\mathrm{b}, \mathrm{c}}$ \\
\hline E. faecium Q (Agri-King) & $5.04^{\mathrm{b}}$ & $4.90^{\mathrm{b}}$ & 8.6 & 8.5 & $50.7^{\mathrm{a}, \mathrm{b}, 5}$ & 97.6 & -1.0 & $67.9^{\mathrm{a}, \mathrm{b}}$ & $25.1^{\mathrm{a}, \mathrm{b}}$ & $7.0^{\mathrm{c}}$ \\
\hline Lactobacillus buchneri (Biotal) & $5.35^{\mathrm{a}}$ & $5.02^{\mathrm{b}}$ & 8.7 & 8.4 & $33.2^{\mathrm{b}}$ & 103.7 & 5.1 & $66.2^{\mathrm{b}, \mathrm{c}}$ & $18.9^{\mathrm{c}}$ & $14.9^{\mathrm{a}}$ \\
\hline L. buchneri 11A44 (Pioneer) & $4.97^{\mathrm{b}}$ & $4.93^{\mathrm{b}}$ & 8.8 & 8.5 & $47.1^{\mathrm{a}, \mathrm{b}}$ & 95.6 & 3.0 & $67.2^{\mathrm{a}, \mathrm{b}, \mathrm{c}}$ & $24.1^{\mathrm{a}, \mathrm{b}}$ & $8.5^{\mathrm{b}, \mathrm{c}}$ \\
\hline L. plantarum and E. faecium 1188 (Pioneer) & $5.00^{\mathrm{b}}$ & $5.00^{\mathrm{b}}$ & 9.0 & 8.5 & $49.5^{\mathrm{a}, \mathrm{b}}$ & 104.7 & 6.1 & $66.9^{\mathrm{a}, \mathrm{b}, \mathrm{c}}$ & $25.0^{\mathrm{a}, \mathrm{b}}$ & $8.1^{\mathrm{c}}$ \\
\hline ARF alone & & $7.43^{\mathrm{a}}$ & & $2.5(5.0)^{4}$ & $<0.5^{\mathrm{c}}$ & 85.0 & 13.6 & $64.2^{\mathrm{c}}$ & $25.6^{\mathrm{a}}$ & $10.3^{\mathrm{b}}$ \\
\hline Standard error & 0.12 & 0.30 & & & 9.9 & 17.9 & & 1.3 & 1.4 & 0.8 \\
\hline \multicolumn{11}{|l|}{ SRF } \\
\hline Control & $5.31^{\mathrm{b}}$ & $5.26^{\mathrm{b}}$ & 6.6 & $7.0(6.9)^{4}$ & $<0.5$ & $198.5^{\mathrm{a}, \mathrm{b}}$ & 108.2 & $52.4^{\mathrm{a}, \mathrm{b}}$ & $35.5^{\mathrm{a}}$ & $12.2^{\mathrm{c}}$ \\
\hline E. faecium $\mathrm{C}$ (Agri-King) & $5.38^{\mathrm{a}, \mathrm{b}}$ & $5.27^{\mathrm{b}}$ & 6.8 & 8.9 & $<0.5$ & $165.3^{\mathrm{a}, \mathrm{b}}$ & 75.0 & $53.1^{\mathrm{a}, \mathrm{b}}$ & $32.4^{\mathrm{b}}$ & $14.5^{\mathrm{b}, \mathrm{c}}$ \\
\hline E. faecium Q (Agri-King) & $5.36^{\mathrm{a}, \mathrm{b}}$ & $5.30^{\mathrm{b}}$ & 6.9 & 6.8 & $<0.5$ & $191.3^{\mathrm{a}, \mathrm{b}}$ & 101.0 & $45.4^{\mathrm{a}, \mathrm{b}}$ & $29.8^{\mathrm{b}, \mathrm{c}}$ & $24.8^{\mathrm{a}, \mathrm{b}}$ \\
\hline L. buchneri (Biotal) & $5.38^{\mathrm{a}, \mathrm{b}}$ & $5.30^{\mathrm{b}}$ & 6.5 & 6.7 & $<0.5$ & $218.2^{\mathrm{a}}$ & 127.9 & $44.3^{\mathrm{b}}$ & $29.2^{\mathrm{b}, \mathrm{c}}$ & $26.5^{\mathrm{a}}$ \\
\hline L. buchneri 11A44 (Pioneer) & $5.43^{\mathrm{a}}$ & $5.32^{\mathrm{b}}$ & 6.5 & 7.1 & $<0.5$ & $156.8^{\mathrm{a}, \mathrm{b}}$ & 66.5 & $44.4^{\mathrm{b}}$ & $29.8^{\mathrm{b}, \mathrm{c}}$ & $25.8^{\mathrm{a}, \mathrm{b}}$ \\
\hline L. plantarum and E. faecium 1188 (Pioneer) & $5.41^{\mathrm{a}, \mathrm{b}}$ & $5.27^{\mathrm{b}}$ & 7.6 & & $<0.5$ & $209.7^{\mathrm{a}, \mathrm{b}}$ & 119.4 & $45.5^{\mathrm{a}, \mathrm{b}}$ & $29.5^{\mathrm{b}, \mathrm{c}}$ & $25.0^{\mathrm{a}, \mathrm{b}}$ \\
\hline $\mathrm{SRF}$ alone & & $5.83^{\mathrm{a}}$ & & $3.6(6.0)^{4}$ & $<0.5$ & $148.8^{\mathrm{b}}$ & 58.5 & $54.1^{\mathrm{a}}$ & $27.2^{\mathrm{c}}$ & $17.8^{\mathrm{a}, \mathrm{b}, \mathrm{c}}$ \\
\hline Standard error & 0.04 & 0.09 & & & & 23.7 & & 3.2 & 1.2 & 4.3 \\
\hline
\end{tabular}

${ }^{a, b, c}$ For each rumen fluid separately, within a column, means with different letters are significantly different $(P<0.05)$.

${ }^{1} \mathrm{pH}-24, \mathrm{pH}-48=\mathrm{pH}$ values after 24 and $48 \mathrm{~h}$ of incubation at $39^{\circ} \mathrm{C}$.

${ }^{2}$ Lactic acid bacteria (LAB) are given as $\log 10 \mathrm{cfu} / \mathrm{mL}$. LAB-24, LAB- $48=\mathrm{LAB}$ after 24 and $48 \mathrm{~h}$ of incubation at $39^{\circ} \mathrm{C} ; \mathrm{Lactic}$ acid- $48 \mathrm{~m} M=\operatorname{lactic}$ acid concentration in $\mathrm{m} M$ after $48 \mathrm{~h}$ of incubation at $39^{\circ} \mathrm{C}$

${ }^{3}$ Total VFA = sum of acetate, propionate, and butyrate in mM. Apparent VFA production is $48 \mathrm{~h}$ total VFA minus time 0 VFA concentrations (mM).

${ }^{4}$ Number in parentheses is from De Man, Rogosa, and Sharpe (MRS) agar.

${ }^{5}$ Large differences between duplicates. 
Table 9. $\mathrm{pH},{ }^{1}$ lactic acid bacteria, ${ }^{2}$ and fermentation products ${ }^{3}$ in autoclaved (ARF) and strained (SRF) rumen fluid with half-milk line corn silages in the fifth experiment.

\begin{tabular}{|c|c|c|c|c|c|c|c|c|c|c|}
\hline \multirow[b]{2}{*}{ umen fluid/silage treatment } & \multirow[b]{2}{*}{$\mathrm{pH}-24$} & \multirow[b]{2}{*}{$\mathrm{pH}-48$} & \multirow[b]{2}{*}{ LAB-24 } & \multirow[b]{2}{*}{$\mathrm{LAB}-48$} & \multirow{2}{*}{$\begin{array}{l}\text { Lactic } \\
\text { acid-48 } \\
(\mathrm{m} M)\end{array}$} & \multirow{2}{*}{$\begin{array}{l}\text { Total } \\
\text { VFA }\end{array}$} & \multirow{2}{*}{$\begin{array}{l}\text { Apparent } \\
\text { VFA } \\
\text { production }\end{array}$} & \multicolumn{3}{|c|}{ VFA $(\mathrm{mol} / 100 \mathrm{~mol})$} \\
\hline & & & & & & & & Acetate & Propionate & Butyrate \\
\hline ontrol & $5.17^{\mathrm{b}}$ & $518^{\mathrm{b}}$ & 84 & & 225 & 1519 & 1025 & 490 & $16 \Omega \Omega^{\mathrm{b}}$ & $347^{\mathrm{a}}$ \\
\hline Lactobacillus plantarum MTD1 (Ecosyl) & $5.37^{\mathrm{a}}$ & $\begin{array}{l}5.18 \\
5.21^{\mathrm{b}}\end{array}$ & $\begin{array}{l}8.4 \\
7.6\end{array}$ & $\begin{array}{l}8.3 \\
8.2\end{array}$ & $\begin{array}{l}22.5 \\
18.6\end{array}$ & $\begin{array}{l}101.9 \\
138.3\end{array}$ & $\begin{array}{r}102.5 \\
88.9\end{array}$ & $\begin{array}{l}49.0 \\
48.2\end{array}$ & $\begin{array}{l}16.2^{\mathrm{b}} \\
15.9^{\mathrm{b}}\end{array}$ & $\begin{array}{l}34.7^{\mathrm{a}} \\
36.2^{\mathrm{a}}\end{array}$ \\
\hline ediococcus pentosaceus (Ecosyl) & $5.21^{b}$ & $5.15^{\mathrm{b}}$ & 8.6 & 8.4 & $38.7^{4}$ & 104.3 & 54.9 & 53.8 & $17.9^{\mathrm{a}, \mathrm{b}}$ & $28.3^{\mathrm{a}, \mathrm{b}}$ \\
\hline plantarum (Agri-King) & $5.27^{\mathrm{a}, \mathrm{b}}$ & $5.05^{\mathrm{b}}$ & 8.1 & 8.1 & $39.4^{4}$ & 111.8 & 62.4 & 52.5 & $19.5^{\mathrm{a}, \mathrm{b}}$ & $28.0^{\mathrm{a}, \mathrm{b}}$ \\
\hline pentosus (Agri-King) & $5.24^{\mathrm{b}}$ & $5.00^{\mathrm{b}}$ & 8.0 & 8.8 & $53.4^{4}$ & 116.8 & 67.4 & 58.4 & $27.3^{\mathrm{a}, \mathrm{b}}$ & $19.4^{\mathrm{a}, \mathrm{b}}$ \\
\hline pentosaceus (Agri-King) & $5.27^{\mathrm{a}, \mathrm{b}}$ & $5.08^{\mathrm{b}}$ & 7.7 & 8.2 & 27.4 & 135.6 & 86.2 & 51.5 & $17.2^{\mathrm{a}, \mathrm{b}}$ & $31.4^{\mathrm{a}, \mathrm{b}}$ \\
\hline $\mathrm{ARF}$ alone & & $7.61^{\mathrm{a}}$ & & $\mathrm{NF}^{5}$ & $<0.5$ & 94.0 & 44.6 & 60.4 & $28.8^{\mathrm{a}}$ & $10.8^{\mathrm{b}}$ \\
\hline tandard error & 0.05 & 0.21 & & & 22.1 & 27.4 & & 6.6 & 4.8 & 8.7 \\
\hline & & & & & & & & & & \\
\hline Control & 5.22 & 5.14 & 4.8 & 5.6 & $<0.5$ & $202.7^{\mathrm{b}}$ & 111.7 & $45.7^{\mathrm{b}, \mathrm{c}}$ & $23.3^{\mathrm{b}}$ & $30.9^{\mathrm{a}}$ \\
\hline plantarum MTD1 (Ecosyl) & 5.26 & 5.12 & 5.7 & 4.4 & $<0.5$ & $237.0^{\mathrm{a}}$ & 146.0 & $43.5^{\mathrm{c}}$ & $25.1^{\mathrm{b}}$ & $31.4^{\mathrm{a}}$ \\
\hline pentosaceus (Ecosyl) & 5.28 & 5.16 & 5.9 & 6.1 & $<0.5$ & $154.3^{\mathrm{c}, \mathrm{d}}$ & 63.0 & $59.0^{\mathrm{a}}$ & $30.3^{\mathrm{a}}$ & $10.8^{\mathrm{c}}$ \\
\hline plantarum (Agri-King) & 5.27 & 5.14 & 5.1 & 5.2 & $<0.5$ & $240.4^{\mathrm{a}}$ & 149.4 & $43.0^{\mathrm{c}}$ & $24.2^{\mathrm{b}}$ & $33.8^{\mathrm{a}}$ \\
\hline pentosus (Agri-King) & 5.30 & 5.06 & 5.9 & 4.3 & $<0.5$ & $184.0^{\mathrm{b}, \mathrm{c}}$ & 93.0 & $42.0^{\mathrm{c}}$ & $22.8^{\mathrm{b}}$ & $35.4^{\mathrm{a}}$ \\
\hline P. pentosaceus (Agri-King) & 5.22 & 5.15 & 6.9 & 5.8 & $<0.5$ & $186.7^{\mathrm{b}, \mathrm{c}}$ & 95.7 & $41.5^{\mathrm{c}}$ & $25.2^{\mathrm{b}}$ & $33.4^{\mathrm{a}}$ \\
\hline SRF alone & & 5.94 & & 4.5 & $<0.5$ & $125.2^{\mathrm{d}}$ & 34.2 & $51.1^{\mathrm{b}}$ & $29.4^{\mathrm{a}}$ & $19.5^{\mathrm{b}}$ \\
\hline tandard error & 0.04 & 0.28 & & & & 12.2 & & 2.2 & 1.2 & 2.5 \\
\hline
\end{tabular}

${ }^{\mathrm{a}, \mathrm{b}, \mathrm{c}}$ For each rumen fluid separately, within a column, means with different letters are significantly different $(P<0.05)$.

${ }^{1} \mathrm{pH}-24, \mathrm{pH}-48=\mathrm{pH}$ values after 24 and $48 \mathrm{~h}$ of incubation at $39^{\circ} \mathrm{C}$.

${ }^{2}$ Lactic acid bacteria (LAB) are given as $\log 10 \mathrm{cfu} / \mathrm{mL}$. LAB-24, LAB-48 = LAB after 24 and $48 \mathrm{~h}$ of incubation at $39^{\circ} \mathrm{C} ; \mathrm{Lactic}$ acid- $48 \mathrm{~m} M=\operatorname{lactic}$ acid concentration in $\mathrm{m} M$ after $48 \mathrm{~h}$ of incubation at $39^{\circ} \mathrm{C}$

${ }^{3}$ Total VFA = sum of acetate, propionate, and butyrate in $\mathrm{m} M$. Apparent VFA production is $48 \mathrm{~h}$ total VFA minus time 0 VFA concentrations (mM).

${ }^{4}$ Large differences between duplicates.

${ }^{5} \mathrm{NF}=$ Not found 
Table 10. $\mathrm{pH},{ }^{1}$ lactic acid bacteria, ${ }^{2}$ and fermentation products ${ }^{3}$ in autoclaved (ARF) and strained (SRF) rumen fluid with half-milk line corn silages in the sixth experiment.

\begin{tabular}{|c|c|c|c|c|c|c|c|c|c|c|}
\hline \multirow[b]{2}{*}{ Rumen fluid/silage treatment } & \multirow[b]{2}{*}{$\mathrm{pH}-24$} & \multirow[b]{2}{*}{$\mathrm{pH}-48$} & \multirow[b]{2}{*}{ LAB-24 } & \multirow[b]{2}{*}{ LAB-48 } & \multirow{2}{*}{$\begin{array}{l}\text { Lactic } \\
\text { acid-48 } \\
(\mathrm{m} M)\end{array}$} & \multirow{2}{*}{$\begin{array}{l}\text { Total } \\
\text { VFA }\end{array}$} & \multirow{2}{*}{$\begin{array}{l}\text { Apparent } \\
\text { VFA } \\
\text { production }\end{array}$} & \multicolumn{3}{|c|}{ VFA (mol/100 mol) } \\
\hline & & & & & & & & Acetate & Propionate & Butyrate \\
\hline \multicolumn{11}{|l|}{ ARF } \\
\hline Control & 5.76 & $5.53^{\mathrm{b}}$ & $7.3(7.3)^{4}$ & $8.5(7.8)^{4}$ & $<0.5$ & $170.0^{\mathrm{a}}$ & 105.4 & $39.2^{\mathrm{b}, \mathrm{c}}$ & 11.9 & 48.8 \\
\hline Enterococcus faecium C (Agri-King) & 5.77 & $5.46^{\mathrm{b}}$ & 6.5 & 8.7 & $<0.5$ & $188.4^{\mathrm{a}}$ & 123.8 & $38.1^{\mathrm{c}}$ & 12.9 & 48.9 \\
\hline E. faecium Q (Agri-King) & 5.77 & $5.27^{\mathrm{b}}$ & 6.7 & 8.4 & 5.0 & $149.8^{\mathrm{a}, \mathrm{b}}$ & 85.2 & $41.1^{\mathrm{b}, \mathrm{c}}$ & 11.7 & 47.1 \\
\hline Lactobacillus buchneri (Biotal) & 5.72 & $5.28^{\mathrm{b}}$ & 7.6 & 7.5 & $<0.5$ & $177.2^{\mathrm{a}}$ & 112.6 & $40.5^{\mathrm{b}, \mathrm{c}}$ & 11.4 & 48.1 \\
\hline L. buchneri 11A44 (Pioneer) & 5.70 & $5.38^{\mathrm{b}}$ & 7.6 & 8.3 & $<0.5$ & $165.0^{\mathrm{a}}$ & 100.4 & $42.4^{\mathrm{b}}$ & 11.5 & 46.1 \\
\hline L. plantarum and E. faecium 1188 (Pioneer) & 5.81 & $5.39^{\mathrm{b}}$ & 8.1 & 8.7 & $<0.5$ & $134.7^{\mathrm{a}, \mathrm{b}}$ & 70.1 & $39.6^{\mathrm{b}, \mathrm{c}}$ & 14.0 & 46.4 \\
\hline $\mathrm{ARF}$ alone & & $7.58^{\mathrm{a}}$ & & $4.0(3.7)^{4}$ & $<0.5$ & $93.1^{\mathrm{b}}$ & 28.5 & $64.2^{\mathrm{a}}$ & 26.0 & 9.7 \\
\hline Standard error & 0.04 & 0.26 & & & & 24.5 & & 1.4 & 1.4 & 1.7 \\
\hline \multicolumn{11}{|l|}{ SRF } \\
\hline Control & $5.42^{\mathrm{a}, \mathrm{b}}$ & $5.28^{\mathrm{b}}$ & $5.0(4.8)^{4}$ & $5.5(5.7)^{4}$ & $<0.5$ & 194.5 & 153.3 & $45.1^{\mathrm{b}}$ & 24.2 & 30.6 \\
\hline E. faecium C (Agri-King) & $5.47^{\mathrm{a}, \mathrm{b}}$ & $5.23^{\mathrm{b}}$ & 6.4 & 6.6 & $<0.5$ & 224.8 & 183.6 & $4.0^{\mathrm{b}}$ & 23.5 & 32.5 \\
\hline E. faecium Q (Agri-King) & $5.39^{\mathrm{a}, \mathrm{b}}$ & $5.17^{\mathrm{b}}$ & 5.7 & 5.5 & $<0.5$ & 206.5 & 165.3 & $45.1^{\mathrm{b}}$ & 26.2 & 28.8 \\
\hline L. buchneri (Biotal) & $5.34^{\mathrm{b}}$ & $5.23^{\mathrm{b}}$ & 5.8 & 5.9 & $<0.5$ & 197.8 & 156.6 & $45.2^{\mathrm{b}}$ & 27.2 & 27.6 \\
\hline L. buchneri 11A44 (Pioneer) & $5.41^{\mathrm{a}, \mathrm{b}}$ & $5.19^{\mathrm{b}}$ & 5.8 & 7.6 & $<0.5$ & 225.9 & 184.7 & $45.3^{\mathrm{b}}$ & 26.6 & 28.1 \\
\hline L. plantarum and E. faecium 1188 (Pioneer) & $5.52^{\mathrm{a}}$ & $5.23^{\mathrm{b}}$ & 6.4 & 7.1 & $<0.5$ & 206.0 & 164.8 & $43.7^{\mathrm{b}}$ & 25.3 & 30.9 \\
\hline SRF alone & & $6.47^{\mathrm{a}}$ & & $3.9(4.2)^{4}$ & $<0.5$ & 120.8 & 79.6 & $53.6^{\mathrm{a}}$ & 25.3 & 21.0 \\
\hline Standard error & 0.06 & 0.09 & & & & 41.0 & & 1.3 & 1.4 & 2.5 \\
\hline
\end{tabular}

a,b,c For each rumen fluid separately, within a column, means with different letters are significantly different $(P<0.05)$.

${ }^{1} \mathrm{pH}-24, \mathrm{pH}-48=\mathrm{pH}$ values after 24 and $48 \mathrm{~h}$ of incubation at $39^{\circ} \mathrm{C}$.

${ }^{2}$ Lactic acid bacteria (LAB) are given as $\log 10 \mathrm{cfu} / \mathrm{mL}$. LAB-24, LAB- $48=\mathrm{LAB}$ after 24 and $48 \mathrm{~h}$ of incubation at $39^{\circ} \mathrm{C} ; \mathrm{Lactic}$ acid- $48 \mathrm{~m} M=\operatorname{lactic}$ acid concentration in $\mathrm{m} M$ after $48 \mathrm{~h}$ of incubation at $39^{\circ} \mathrm{C}$.

${ }^{3}$ Total VFA = sum of acetate, propionate, and butyrate in $\mathrm{mM}$. Apparent VFA production is $48 \mathrm{~h}$ total VFA minus time 0 VFA concentrations (mM).

${ }^{4}$ Number in parentheses is from De Man, Rogosa, and Sharpe (MRS) agar. 
included in silage inoculants could survive under rumenlike conditions. A previous study (Weinberg et al., 2003, 2004 ) indicated that the LAB in freeze-dried silage inoculants survived in $\mathrm{RF}$ in vitro during 72 to $96 \mathrm{~h}$ of incubation at $39^{\circ} \mathrm{C}$. In $\mathrm{SRF}$, they resulted in higher $\mathrm{pH}$ values than those in uninoculated controls. The objective of the present study was to follow the passage of LAB from inoculated and control silages to $\mathrm{RF}$ in vitro and to determine whether they survived and how they affected the rumen environment. The ARF was used to determine whether LAB from silage were introduced into the RF in the absence of competition; the SRF was used to follow possible interactions between the endogenous rumen microorganisms and the LAB from the silages. The ratio of silage to $R F$ (1:10) was based on the ratio that results from the amount of silage in the ration for dairy cows in Israel (about $20 \mathrm{~kg}$ wet weight and rumen volume of $150 \mathrm{~L}$ ). Such an addition should have enriched the RF in LAB by $10^{5}$ to $10^{7} \mathrm{LAB} \mathrm{cfu} / \mathrm{mL}$, according to the LAB populations in the silages (Tables 1 to 3 ). The LAB numbers reveal that inoculated silages did not necessarily contain more LAB than the control silages. The moist early dent corn silage (190 g/kg DM) was used along with the drier silages to provide a wide range of silage DM for this study. In that context, experiments in Northern Ireland showed that feeding moist grass silages treated with $L$. plantarum MTD1 enhanced ruminant performance (Keady et al., 1994, Keady and Steen, 1994, 1995). Therefore, we wanted to include a moist silage in our study.

To ensure that large numbers of the specific inoculant strains were transferred from the silages into the $\mathrm{RF}$ during incubation, we applied a high inoculation rate in our study $\left(10^{6} \mathrm{cfu} / \mathrm{g}\right.$ of forage). The present results indicate that in vitro $\mathrm{LAB}$ were transferred from the silage into the $\mathrm{RF}$ and that their numbers even increased during the $48 \mathrm{~h}$ of incubation. We assumed that the LAB found in the RF samples belonged to the strains that were present in the silage samples. Such an assumption could be verified by tagging the target strains with some distinctive property such as resistance to antibiotics, or by using molecular biological tools such as PCR $16 \mathrm{~S}$ rDNA sequencing (Tannock, 2003).

The LAB numbers in ARF were close to those expected based on the amount of silage added, but those in the $\mathrm{SRF}$ were smaller than those in the ARF, probably because of competition with the endogenous populations in SRF. No consistent differences were observed between the effects of the addition of uninoculated control silages and those of the various inoculated silages, with regard to $\mathrm{pH}$ and LAB numbers in the RF. The lactic acid found in the $\mathrm{ARF}$ could have originated directly from the silage samples or from fermentation of the added glucose by the LAB in the RF. The theoretical lactic acid concentra- tion in the $\mathrm{RF}$ originating from the silages, based on the lactic acid content in the silage samples, varies between 12 and $26 \mathrm{mM}$. The rest of the lactic acid must have been produced from the glucose by silage LAB in the RF. In a previous study with freeze-dried LAB cultures, some lactic acid that was found in clarified rumen fluid had been produced by the LAB in the RF (Z. G. Weinberg, unpublished data, 2003).

In the trials with corn silages, after $48 \mathrm{~h}$ of incubation the $\mathrm{pH}$ values of the $\mathrm{SRF}$ without silage addition (SRF alone) were higher than those of the silage-added SRF. This observation is not consistent with the results obtained previously by adding freeze-dried LAB to SRF (with or without glucose); in these studies the RF with $\mathrm{LAB}$ had higher $\mathrm{pH}$ values than those of the control RF without added LAB (Weinberg et al., 2003, 2004). Buffering the rumen $\mathrm{pH}$ could be one mode of action by which silage inoculants enhance the functionality of specific rumen microorganisms, especially in cases where the $\mathrm{pH}$ decreases following high-energy feeding (Weimer, 1996). It would be worthwhile to find out the conditions under which LAB silage inoculants result in higher $\mathrm{pH}$ in $\mathrm{RF}$.

In the previous study with silage inoculants LAB in RF (Weinberg et al., 2003), L. plantarum MTD1 resulted in the highest concentrations of VFA in SRF relative to other inoculants. The current results do not identify any silage treatment (inoculants or control) that had a consistent effect on total VFA content, apparent VFA production, VFA molar fraction, or lactic acid content in the RF.

Finally, the published literature indicates that probiotic effects can be attributed to specific microbial strains. Cattle clearly benefited from being fed with grass silage inoculated with L. plantarum MTD1 (Keady et al., 1994, Keady and Steen, 1994, 1995). However, the current data are not yet sufficient to identify a silage inoculant that might exert beneficial probiotic effects in ruminants. More research is needed to study the interactions between feeding status of the animal and the effect of LAB silage inoculants on animal performance, and the interactions between LAB from silage and rumen microorganisms and fiber digestibility. Such information will maximize beneficial responses in silage preservation and in animal performance.

\section{ACKNOWLEDGMENTS}

This research was supported by Research Grant No. IS-3297-02 from BARD, The United States-Israel BiNational Agricultural Research and Development Fund.

Contribution from the Agricultural Research Organization, The Volcani Center, Bet Dagan, Israel, No. 403/ 04 series. 


\section{REFERENCES}

Barker, S. B., and W. H. Summerson. 1941. The colorimetric determination of lactic acid in biological material. J. Biol. Chem. 138:535-554.

Fuller, R. 1989. Probiotics in man and animals. J. Appl. Bacteriol. 66:365-378.

Keady, T. W. J., and W. J. Steen. 1994. Effects of treating low dry matter grass with a bacterial inoculant on the intake and performance of beef cattle and studies on its mode of action. Grass Forage Sci. 49:438-446.

Keady, T. W. J., and W. J. Steen. 1995. The effects of treating low dry matter, low digestibility grass with a bacterial inoculant on the intake and performance of beef cattle and studies on its mode of action. Grass Forage Sci. 50:217-226.

Keady, T. W. J., and W. J. Steen. 1996. Effects of applying a bacterial inoculant to silage immediately before feeding on silage intake, digestibility, degradability and rumen volatile fatty acids concentrations in growing beef cattle. Grass Forage Sci. 51:155-162.

Keady, T. W. J., W. J. Steen, D. J. Kalpatrik, and C. S. Mayne. 1994. Effects of inoculant treatment on silage fermentation, digestibility and intake by growing cattle. Grass Forage Sci 49:284-294.

Khuntia, A., and I. C. Chaudhary. 2002. Performance of male cross bred calves as influenced by substitution of grain by wheat bran and the addition of lactic acid bacteria to diet. Asian-Australas. J. Anim. Sci. 15:188-194.
Kung, Jr., L., M. R. Stokes, and C. J. Lin. 2003. Silage additives. Pages 305-360 in Silage Science and Technology, D. R. Buxton, R. E. Muck and J. H. Harrison, eds. Am. Soc. Agronomy, Inc., Madison, WI.

Malik, R., and D. D. Sharma. 1998. In vitro evaluation of different probiotics as feed supplement. Indian J. Dairy Sci. 51:357-362.

Muck, R. E. 1993. The role of silage additives in making high quality silage. Pages 106-116 in Silage Production from Seed to Animal, NRAES-67, Northeast Regional Agricultural Engineering Service, Syracuse, NY.

Salawu, M. B., E. H. Warren, and A. T. Adesogan. 2001. Fermentation characteristics, aerobic stability, and degradation of ensiled pea/ wheat bi-crop forages treated with two microbial inoculants, formic acid or quebracho tannins. J. Sci. Food Agric. 81:1263-1268.

Tannock, G. W. 2003. Probiotics and prebiotics, where are we going? Pages 1-40 in Probiotics and Prebiotics, Where Are We Going? G.W. Tannock, ed. IBT Global, Barking, London, UK.

Weimer, P. J. 1996. Why don't ruminal bacteria digest cellulose faster? J. Dairy Sci. 79:1496-1502.

Weinberg, Z. G., and R. E. Muck. 1996. New trends in development and use of inoculants for silage. FEMS Microbiol. Rev. 19:53-68.

Weinberg, Z. G., R. E. Muck, and P. J. Weimer. 2003. The survival of lactic acid bacteria in rumen fluid. J. Appl. Microbiol. 94:10661071.

Weinberg, Z. G., R. E. Muck, P. J. Weimer, Y. Chen, and M. Gamburg. 2004. Lactic acid bacteria used in silage inoculants as probiotics for ruminants. Appl. Biochem. Biotechnol. 118:1-10. 